\title{
SUÁREZ ET DIEGO MAS (1553-1608). LA MÉTAPHYSIQUE RESTITUÉE À ELLE-MÊME PAR LE RENOUVELLEMENT DE LA COMPRÉHENSION DE L'ÉTANT
}

\author{
SUAREZ AND DIEGO MAS (1553-1608). THE METAPHYSICS \\ RESTORED TO ITSELF BY RENEWING THE \\ UNDERSTANDING OF BEING
}

\author{
JeAn-Paul Coujou \\ Institut catholique de Toulouse \\ Institut Michel Villey, Paris
}

\section{RESUME / RESUMEN}

Avec les œuvres de Diego Mas et Suárez, un projet commun est affirmé à partir de la mutation décisive des concepts ontologiques légués par la tradition et consacrée par l'avènement d'une métaphysique transcendantale recentrant le processus de la production du savoir sur l'universel. Le point de départ d'un renouvellement du questionnement métaphysique requiert l'examen systématique de son historicité, ce qui explique précisément que s'il est impossible de faire l'économie de l'héritage fondateur d'Aristote, il est désormais nécessaire de reconnaître que l'histoire de la métaphysique ne saurait s'y réduire.

Mots clés / Palabras clave: Dieu, étant, métaphysique, raison, science, théologie, transcendantal, universel 


\section{ABSTRACT}

With the works of Diego Mas and Suarez, a common project is affirmed from the decisive mutation of the ontological concepts handed down by tradition and consecrated by the advent of transcendental metaphysics refocusing the process of production of knowledge on the universal. The starting point of a renewal of metaphysical questioning requires systematic examination of its historicity, which explains precisely that if it impossible to do without the founding heritage of Aristotle, henceforth it is necessary to recognize that metaphysic's history cannot be reduce to it.

Keywords: God, being, metaphysics, reason, science, theology, transcendental, universal

Diego Mas a fait paraître en 1587 à Valence, dix années avant les Disputes métaphysiques de Suárez ${ }^{1}$ et deux ans après les Commentaria in Primam Partem Divi Thomae (1585) de Domingo Báñez ${ }^{2}$, son premier livre qui est également son ouvrage majeur: Dispute métaphysique sur l'étant et ses propriétés (Metaphysica disputatio de ente et eius proprietatibus quae communi nomine inscribitur de transcendentibus $)^{3}$. Tout porte à penser, au regard de ces trois publications centrales réalisées à la fin du $\mathrm{XVI}^{\mathrm{e}}$ siècle, que l'on assiste à un retour de 1'enseignement de la métaphysique dans les universités espagnoles (très précisément à Valence et à Salamanque) jusqu'alors centrées sur des disciplines comme la logique, la dialectique ou la philosophie naturelle. Or, ce retour requiert un recentrage sur ce que peuvent et doivent être l'objet et la finalité de la métaphysique pour qu'elle soit en mesure de faire prévaloir sa spécificité et son autonomie, notamment relativement à la dialectique et à la physique.

Une ambiguïté et une difficulté préalables en résultent nécessairement pour Diego Mas. En effet, d'une part, aucun projet de recherche métaphysique ne peut prétendre faire l'économie de l'héritage aristotélicien sous peine d'errer et

1 Francisco Suárez, Metaphysicorum Disputationum, in quibus et universa naturalis theologia ordinate traditur, et quaestiones omnes ad duodecim Aristotelis libros pertinentes accurate disputantur. Tomus prior. Autore R.P. Francisco Suárez e Societate Iesu ...Tomus Posterior ... (Salmanticae, apud Ioannem et Andream Renaut fratres, 1597).

2 Domingo Báñez, Scolastica commentaria in primam partem ... D. Thomae usque ad LXIII quaestionem complectentia (Venetiis, 1585).

3 Diego Mas, Disputación metafisica sobre el ente y sus propiedades (1587), Traducción y introducción de J. Gallego Salvadores, (Pamplona, EUNSA, 2003). 
de ne pas s'assurer un point de départ ferme pour ses investigations. D'autre part, il faut faire le constat à partir des critiques de l'École de Salamanque et de celles de l'humanisme de la Renaissance, de la décadence de l'esprit scolastique liée à l'assujettissement doctrinal à la pensée d'Aristote, contraignant à ne reconnaître pour la raison d'autre voie que la conformité à un modèle qu'il ne serait possible ni de modifier, ni d'améliorer. Cette ambiguité s'accompagne d'une difficulté : refonder la légitimité de la métaphysique en en garantissant le caractère systématique et universel tout en délimitant clairement son champ d'exercice au regard des autres disciplines composant le champ d'étude de la philosophie. Pour ce faire, il est nécessaire, ainsi que le précise Diego Mas dans le prologue à son œuvre 4 , de renverser la priorité quant à l'ordre à adopter dans l'apprentissage du savoir philosophique. En effet, si l'on considère la dialectique et la théologie sacrée, on constate qu'il est problématique de parvenir dans le processus de leur acquisition à une connaissance claire et distincte de leur objet ; cela condamne non seulement à ne pas être en mesure d'établir un lien rigoureux entre les différentes disciplines constituant la sagesse, mais également à procéder de manière désordonnée et en étant privés de la lumière naturelle de la raison.

Il existe pourtant un point de départ sûr : la connaissance de l'étant et de ses propriétés transcendantales ; c'est elle qui ouvre le chemin pour accéder de manière exacte et " digne $»^{5}-$ c'est-à-dire qui en fonde légitimement la valeur - à l'ensemble des sciences. Tel est pour Diego Mas le point d'Archimède destiné à assurer une reconstruction de l'ordre du savoir. De la position de l'étant universellement considéré découleront l'examen et la détermination de l'essence et de l'existence, de l'unité, de la vérité et de la bonté . Ces propriétés transcendantales désignant certains prédicats excédant les catégories aristotéliciennes s'accordent à la totalité des choses, ces dernières y étant nécessairement renvoyées dans le processus de détermination de leur spécificité ou de ce que l'on pourrait qualifier selon une terminologie heideggérienne, d'étantité de l'étant. Cela requiert néanmoins: $1^{\circ}$ ) une connaissance approfondie de l'histoire de la théologie et de la métaphysique ${ }^{7}, 2^{\circ}$ ) de montrer en quoi les traités de Chrysostome Javelli ${ }^{8}$ Sur les transcendantaux et l'opuscule

$4 \quad$ Mas, Disputación metafisica, 419.

5 Mas, Disputación metafisica, 419.

6 Respectivement livres II à V des Disputes.

7 Mas, Disputación metafisica, 419. Diego Mas se réfère notamment à Albert le Grand, Thomas d'Aquin, Jean Duns Scot, Jean Capréolus, Jean Jandun, Cajetan, Sylvestre de Ferrare ...

8 Crysostomus Iavellus, Tractatus de Transcendentibus previus methaphisice (s.e.: 1528). 
de Pic de la Mirandole Sur l'étant et l'un ${ }^{9}$, même s'ils ont le mérite d'ouvrir la voie dans une telle recherche, ne se donnent pas les moyens de répondre à leur ambition, $3^{\circ}$ ) un effort constant à la fois dans la conduite incessante d'un questionnement systématique - celle de la dispute, méthode léguée par la tradition scolastique - et d'explicitation linguistique ${ }^{10}$ sans le préalable de laquelle l'entendement humain est soumis à la fois à la confusion et à l'impossibilité de mener à son terme sa démarche. Pour ce faire, il faut prendre exemple sur l'impératif de clarté devant guider l'exposition du savoir métaphysique, propre au style adopté par les écoles stoïciennes ${ }^{11}$, 1 'Académie et les Péripatéticiens ${ }^{12}$. Conformément à l'esprit d'une scolastique réformée dans la seconde moitié du $\mathrm{XVI}^{\mathrm{e}}$ siècle, toute question en métaphysique doit être formulée selon trois moments : l'énonciation claire et distincte, la réfutation des doctrines erronées et l'exposition de la vérité, la réponse aux objections et aux doutes.

\section{LE RENOUVELLEMENT DE LA RECHERCHE METAPHYSIQUE}

Tant pour Diego Mas que pour Suárez, il convient, pour répondre à la crise de légitimité de la scolastique, de revenir à une lecture rigoureuse d'Aristote qui, $1^{\circ}$ ) tout en posant $1^{\prime} œ u v r e$ de ce dernier comme moment fondateur dans l'histoire de la métaphysique, n'en demeure pas pour autant assujettie à son autorité, et $2^{\circ}$ ) l'inscrit à partir de la référence à la tradition, dans la constitution désormais nécessaire d'une histoire métaphysique de la philosophie première. Ce retour est porteur d'une innovation destinée à opérer une différenciation concernant le champ de la constitution des différents savoirs dans le système philosophique qui ne saurait se réduire à la distinction traditionnellement invoquée entre la dialectique et la métaphysique. Il s'agit d'une restitution, celle

9 Jean Pic de la Mirandole, L'être et l'un in CEuvres philosophiques (Paris: PUF, 1995), Traduction par O. Boulnois \& G. Tognon.

10 Mas, Disputación metafisica, Prologue, 420.

11 Mas Disputación metafisica, mentionne p. 420, les Tusculanes et les Académiques de Cicéron, les Questions naturelles de Sénèque.

12 Alexandre d'Aphrodise, Quaestiones naturales et morales ..., (Venetiis, s.e. 1546); Ioannes Philoponus, Aristotelis de anima libri tres ..., (Lugduni, s.e. 1544) (Jean Philopon, Commentaire sur le traité « De l'âme » d'Aristote (vers 510-515), édition par Michael Hayduck, In Aristotelis « De Anima » libros commentaria, Berlin: De Gruyter, 1897), et Simplicius, In libros de Anima Aristotelis, interprete I. Fasseolo Patavino (Venetiis, s.e. 1543), Simplicius, De anima, édition par Michael Hayduck, In Aristotelis « De anima », coll. « Commentaria in Aristotelem Graeca » (CAG), t. XI, (Berlin: De Gruyter, 1882). 
de la place légitime de la métaphysique, qui ne peut être effectuée que par une redéfinition préalable de son statut scientifique et de sa finalité.

Dès lors, pour les deux auteurs, il faut créer les conditions de l'élaboration d'une métaphysique systématique sans laquelle toute rénovation de la scolastique sera vouée à l'échec pour, dans un deuxième temps, délimiter le champ d'exercice légitime des savoirs constitutifs de la sagesse philosophique. Ainsi que le résume Suárez, pour apporter une justification au fait d'avoir interrompu momentanément ses commentaires théologiques sur l'œuvre de Thomas d'Aquin, il devient impératif d'" accorder, ou mieux, restituer à la doctrine métaphysique le lieu et la place qui lui reviennent. " ${ }^{13} \mathrm{La}$ réinterprétation du préfixe "méta » y participe précisément en invitant à la recomposition intellectuelle de son objet qui requiert de prendre en considération, par la « dispute », le fait selon lequel la métaphysique dénature ou dénie son contenu authentique lorsqu'elle se réduit à un commentaire ou pire à une paraphrase de la Métaphysique d'Aristote. Le défi à relever pour les deux auteurs est le suivant : expliciter le domaine d'exercice de la métaphysique afin que, d'une part, le projet initial qui lui garantit sa raison d'être, « la connaissance des premiers principes et des premières causes» ne soit pas mis entre parenthèses ou dénaturé, par la multiplicité des commentaires qui en a résulté. D'autre part, l'encyclopédie des savoirs philosophiques se décompose en quatre champs spécifiques: la métaphysique, la dialectique, la rhétorique et la physique; et si la métaphysique a une place à part, c'est parce qu'il lui revient de développer une théorie universelle de l'étant, d'en établir le caractère connaissable, tout en explicitant l'objet des différentes disciplines dans leur liaison aux genres de réalité.

La création d'une métaphysique autonome dans le champ du savoir philosophique s'avère par conséquent indissociable, au regard de la méthode qu'elle met en œuvre, de sa systématicité. Il devient désormais nécessaire, dans le processus de position de la systématicité de la métaphysique, d'établir qu'elle n'est pas réductible, dans son déploiement, au seul traitement de questions spécifiques aux enjeux déjà délimités par la tradition des commentaires sur la philosophie aristotélicienne. Cet objectif, indispensable à la formation de l'entendement humain, ainsi qu'aux règles devant présider à sa bonne marche, ne peut que s'avérer utile pour Suárez, tant pour la théologie que pour les autres

13 Franciscus Suárez, Opera Omnia, Volume 25, In Metaphysicam Aristotelis. Disputationes metaphysicae, (I-XXVII), (Paris : Vives 1856), Ratio et discursus totius operis, Ad lectorem, $1^{\circ} \S$, sans pagination. 
sciences $^{14}$, alors que pour Diego Mas, une telle utilité concernera également l'exercice de la dialectique, de la philosophie et de la théologie ${ }^{15}$. Un dénominateur commun propre à ces deux démarches s'en dégage: l'explicitation d'une science première et universelle portant sur l'étantité dont il faut poser l'univocité ; ainsi, la métaphysique fait de l'appréhension de l'étantité de l'étant dans son abstraction la plus extrême la condition de possibilité de son universalité. Elle doit par là même se distinguer des metaphysicae speciales dont l'objet est particulier. Restituer la métaphysique à elle-même, implique dès lors une distinction entre une metaphysica generalis portent sur l'étant et les métaphysiques spéciales portant sur des objets dissociés du sensible. Tant pour Diego Mas que pour Suárez, il s'agit de montrer qu'il ne pourra y avoir de renouvellement de la métaphysique qu'à dépasser la réduction de son objet à une classe particulière d'étants.

L'exigence commune d'une métaphysique systématique chez les deux auteurs requiert la dispute comme méthode d'exposition, même si l'on peut constater une divergence formelle dans le procédé utilisé. En effet, pour le premier, il appartient au style de la dispute d'exposer les différentes thèses et raisons qui la légitiment, afin ensuite, par une démarche analytique du problème formulé, en dégager un ensemble de conclusions, tout en répondant aux objections des contradicteurs. Quant à Suárez, il se conforme à l'héritage de la méthode scolastique indissociable dans son esprit de la disputatio universitaire, c'est-à-dire du procédé consistant à décider par preuve, en faisant usage de la technique de la question, qu'il s'agisse selon le modèle de la première scolastique des questions disputées ou de questions extraordinaires qualifiées de questions quodlibétales. L'exposition des thèses s'inscrivant dans une exigence de synthèse de l'histoire de la métaphysique a pour finalité la résolution du problème initial et des apories mentionnées au cours des sections composant la dispute. La présentation d'une métaphysique systématique ne dissocie pas, dans son développement, l'ordre des raisons de connaître de l'ordre des raisons d'être, présentant, par voie de conséquence, le travail permanent de la pensée sur ellemême dans son lien indéfectible avec la question de l'être. Originellement, la dispute est identifiable à une méthode d'apprentissage et à un exercice de confrontation critique à l'histoire de la tradition; elle porte par la révélation de la dimension de vérité susceptible d'être présente dans la thèse adverse, une condition de l'émergence du vrai. Chez les deux auteurs, l'alternance systématique des objections, des exposés et des réfutations est indissociable de

15 Diego Mas, Disputación metafísica, Préface, 419. 
l'acte de la pensée en débat avec elle-même et confrontée à un ordre donné par la raison.

Néanmoins, si un tel ordre ne peut être révélé que par la raison, cette dernière le découvre hors d'elle-même. En fin de compte, il appartient à la dispute, en répondant à un impératif d'ordonnancement systématique du savoir de rappeler, lors du travail de la pensée que l'ordre réel n'est pas différent de l'ordre de la raison. Dans la dispute, qui respecte les règles formelles de la pensée cohérente, le travail de la raison se traduit par les opérations de l'intellect, à savoir : $1^{\circ}$ ) la constitution des concepts permettant l'établissement d'une classification des étants selon leurs différences intelligibles, $2^{\circ}$ ) la formulation de jugements impliquant l'énonciation de propositions composées de concepts considérées comme vraies parce qu'en adéquation avec ce qu'elles disent, $3^{\circ}$ ) la mise en œuvre, par la raison discursive, de raisonnements ayant pour fonction de relier des propositions pour qu'on soit en mesure de manière déductive d'engendrer un enchaînement de vérités. Cependant, au regard du statut de la dispute, s'il revient à la métaphysique au moyen de ce procédé de manifester l'essence de la chose et, par conséquent, de déterminer la nature exacte de la définition, on ne saurait pour autant confondre cette même métaphysique à un pur procédé argumentatif. En opposition à la dialectique assimilée à un art de la connaissance du fait qu'elle en fournit les instruments ${ }^{16}$ et examine le mode de conception et d'intelligibilité de la division des choses, la métaphysique, en tant que science spéculative, étudie les distinctions des choses telles qu'elles sont en elles-mêmes.

La proximité entre Diego Mas et Suárez concernant la méthodologie employée, ne saurait passer sous silence une différence dans l'exposition du contenu qui ne peut être réduite au constat évident d'une différence d'amplitude entre ces deux encyclopédies métaphysiques ; cela peut s'expliquer notamment parce que la recherche de la vérité propre au projet suarézien, s'accompagne d'un débat constant avec l'histoire de la philosophie, indissociable de l'exposition d'une histoire métaphysique de la métaphysique. Il ne s'agit pas seulement de refonder l'objet de la métaphysique, il faut, pour Suárez, rendre indissociable cette exigence d'une généalogie et d'un développement historique de la question métaphysique. Concernant le rapport au contenu, il convient d'avoir à l'esprit, conformément à l'héritage aristotélicien, que la possibilité de la science n'est envisageable que si elle porte sur quelque chose qui est, étant donné qu'il ne peut y avoir de science de ce qui n'est pas. Par conséquent, les 
disciplines que l'on qualifie de sciences ne constituent pas tant une connaissance de l'étant que d'un étant particulier ou d'un genre de l'étant, c'est-à-dire d'une région circonscrite de l'étant. C'est pourquoi il doit y avoir une science de l'étant en tant qu'étant ou de l'étant comme tel, à savoir du fait qu'il est purement et simplement un étant.

Si l'on considère la position de Diego Mas au regard de cette perspective, il opère, par la méthodologie qu'il adopte, une tripartition du savoir métaphysique destinée à rendre raison du fonctionnement de l'ensemble des sciences. Un tel savoir, obéissant au principe selon lequel il faut, en premier lieu, exposer ce qui est le plus commun et le plus universel ${ }^{17}$, implique : $1^{\circ}$ ) une détermination extensive et universelle de l'étant, $2^{\circ}$ ) une explicitation synthétique de ses principes, $3^{\circ}$ ) une exposition analytique de ses propriétés transcendantales. L'œuvre monumentale de Suárez dans sa composition en 54 disputes se présente, quant à elle, sous une forme bipartite. Dans un premier temps, correspondant au premier volume, il s'agit de déterminer l'étant selon la totalité de son amplitude en en explicitant les propriétés et les causes. La théorie de la causalité des diverses manifestations de l'étant qui clôt ce moment met en place la compréhension de la division de l'étant en infini et fini qui est posée comme 《la division première et la plus essentielle de l'étant. $\rangle^{18}$ Le deuxième moment est consacré aux objets métaphysiques pouvant être élaborés par notre raison et notamment l'étant premier, Dieu, pour aboutir à une compréhension catégoriale systématique de l'étant. Le partage entre l'infini et le fini est au fondement du mouvement de la pensée consistant à « descendre vers les raisons définies des étants $\gg{ }^{19}$ dans le but de rendre intelligible par la détermination catégoriale (substance/accident, qualité, puissance, action, passion ...) leurs multiples divisions.

L'étant réel, objet de la métaphysique pour Suárez, qu'il soit fini ou infini, se caractérise par son " aptitude à exister », ce qui l'identifie à un étant possible antérieurement à toute référence à la nécessité ou à la contingence. C'est à partir d'une telle aptitude qu'il devient légitime d'établir ce qu'il y a de commun entre la créature et le Créateur. Ens désigne un concept d'une simplicité extrême qui, par son absolue priorité, s'impose à notre intellect; il exprime ce qui peut être ou ce qui est possible réellement. Pour Diego Mas, il est identifiable à l'étant

17 Mas, Disputación metafísica, Livre I, Ch. VI, n. 66, 437.

18 Suárez, Opera Omnia, Volume 26, Disputationes metaphysicae (XXVIII-LIV), (Paris: Vives 1856),

Introduction, 1.; Suárez, Opera Omnia, Volume 25, 8, n. 1, 137.

19 Suárez, Opera Omnia, Volume 25, 8, n. 1, 137. 
qui a une réalité indépendamment des opérations de l'intellect, par différence à l'étant de raison qui n'a d'existence que par une production de l'entendement. ${ }^{20}$ Il est ce qui a un être dans l'intellect comme objet connu. ${ }^{21}$ Or, pour la métaphysique, traiter de l'étant comme étant réel, signifie tout aussi bien se référer au Créateur, premier de tous les étants, qu'aux substances immatérielles et aux créatures comme étants finis ${ }^{22}$. Pour les deux auteurs, il convient dès lors d'excéder la réduction de l'objet de la métaphysique à une classe particulière d'étants, quelle qu'elle soit, tout en arguant que la théologie naturelle, comme discours sur Dieu, étant suprême, ne peut être amenée à sa compréhension achevée pour l'intellect humain qu'à partir des créatures. Cependant, il faut également préciser que la science de l'étant ne trouve son accomplissement dans le savoir philosophique qu'à partir de la fondation de l'existence en acte de ce même étant dans l'acte pur d'exister qu'est l'Ipsum Esse. "S'il est impossible d'être un bon théologien sans avoir préalablement établi les fondements fermes de la métaphysique ${ }^{23}$, il apparaît également que c'est dans la théologie que la science de l'étant trouve son aboutissement si l'on entend la philosophie comme processus globalisant exprimant un effort spéculatif pour rendre intelligibles les étants en tant qu'ils sont, sans se limiter à la question de savoir s'ils sont ceci ou cela, s'ils ont telle ou telle essence.

\section{L'OBJET PRINCIPAL ET L'OBJET ADEQUAT DE LA METAPHY- SIQUE}

La refondation de l'objet de la métaphysique met en œuvre un renversement ouvrant la voie à l'émergence de l'ontologie en tant que connaissance spécifique et non plus dérivée. En effet, il n'appartient plus à la science de l'universel d'être réglée par la science du premier, il faut bien plutôt, afin de rendre effective cette ambition de renouveau, poser que la science de l'universel englobe la science du premier ${ }^{24}$. Il en découle que le Créateur, étant suprême, est partie intégrante de l'objet de la métaphysique comme objet premier, sans pour autant être assimilable à son objet adéquat ${ }^{25}$. Par conséquent, la priorité dans l'ordre de l'être n'est pas transposable dans l'ordre de la connaissance. Et la distinction pagination.

24 Suárez, Opera Omnia, Volume 25, n. 9, 24-25.

25 Suárez, Opera Omnia, Volume 25, 1, n. 11, 5.
Mas, Disputación metafísica, Livre I, Ch. IV, n. 35, 430.

Mas, Disputación metafisica, Livre I, Ch. IV, n. 42, 431.

Suárez, Opera Omnia, Volume 25, 1, n. 26, 11.

Suárez, Opera Omnia, Volume 25 , Ratio et discursus totius operis, Ad lectorem, $1^{\circ} \S$, sans 
effectuée entre l'objet principal et l'objet adéquat crée les conditions d'une émergence d'une science de l'étant (ou d'une ontologie qui n'a pas encore trouvé son nom) mettant en œuvre une universalisation possible de l'objet de la métaphysique. Tout se passe comme si l'absence de dénomination résultait d'un moment d'indécision qui, en tranchant le nœud gordien concernant dans l'ordre des priorités l'articulation entre l'objet de la métaphysique et celui de la théologie naturelle, exprimait le fait d'être pris de court face à la désignation à assigner à la discipline issue d'un tel geste de rupture. Comment et à quelles conditions penser une science de l'étant pour qu'elle puisse s'achever autrement que dans une théologie, ou pour qu'elle puisse légitimement se concevoir en s'autonomisant dans le processus de la connaissance de ce qui serait qualifié de science du divin ? L'effort pour y répondre suppose que l'on prenne désormais acte de la division du point de départ de la métaphysique en metaphysica generalis portant sur la connaissance universelle et abstraite de l'étant et en metaphysica specialis subdivisée en théologie, cosmologie et psychologie ${ }^{26}$. Dès lors, pour Suárez, l'organisation du savoir de l'étant comme étant réel en s'articulant à une telle distinction, ouvre la voie à une théorie de la connaissance qui, en reposant sur l'unité du concept d'étant et sur la reconnaissance que l'universel ne peut être accessible aux sens, ne manquera pas de questionner les pouvoirs de l'expérience, l'exercice légitime du jugement, les limites de l'intellect et les pouvoirs du langage. Il est par conséquent impératif de procéder, pour mener à bien le projet de la dispute métaphysique, à une délimitation de la puissance ou faculté cognitive tout en traçant le cadre d'une science de l'entendement.

Ainsi, comme l'explicite Diego $\mathrm{Mas}^{27}$, il y a une relation comparable entre l'étant et ses propriétés et l'âme rationnelle et ses facultés. Les puissances de l'âme rationnelle sont au nombre de trois : l'intellect, la mémoire et la volonté. Il en est de même pour les propriétés de l'étant : l'un, le vrai et le bien. Il existe une correspondance : $1^{\circ}$ ) entre l'un et la mémoire par laquelle une unification de la diversité de ce qui s'est produit est possible ; $2^{\circ}$ ) entre le vrai et l'intellect puisque la conformité de ce dernier aux étants est la condition de la vérité $; 3^{\circ}$ ) entre le bien et la volonté qui se porte vers le premier en tant qu'il est appétable.

Néanmoins, pour qu'une telle relation entre l'étant et ses propriétés revête de la pertinence et du sens, il convient pour Diego Mas de prendre la mesure de la portée des mots et des limites qu'il est nécessaire d'opposer à leur usage. Ainsi, dans le langage, une subdivision s'impose entre les mots signifiant des 
natures concrètes, à savoir des attributs liés à un sujet, et ceux exprimant des natures abstraites, c'est-à-dire des attributs séparés du sujet ${ }^{28}$. Les termes abstraits désignent une nature pure et sans mélange. Relativement à cette distinction, « étant » (ens) correspond à un nom concret alors que « être » (esse) renvoie à un nom abstrait. On retrouve ainsi les mêmes différences entre l'ordre du concret et de l'abstrait qu'entre l'ordre de l'étant et celui de l'être. L'usage concret du langage signifie que l'homme habite dans l'étant, l'usage abstrait du langage l'invite à dire l'être de cet étant et à dire par là même l'être de cet étant qui dit l'étant ${ }^{29}$. Dès lors, l'être implique, par son abstraction, qu'il est tel par lui-même et qu'il ne reçoit pas l'être d'autre chose puisqu'il l'a en lui-même et par lui-même. Quant à l'étant, il est par participation à l'être lui-même. Il faut également ajouter que « l'être constitue une certaine partie de l'étant et que ce dernier constitue un tout car il est composé de la forme de l'être et du sujet qui est. $\gg{ }^{30} \mathrm{Il}$ est légitime d'en conclure que l'être n'est entaché par aucune nature distincte de lui, alors que l'étant se caractérise par de nombreuses propriétés distinctes de sa nature. Ainsi, l'essence de l'homme renvoie à sa nature sans mélange ou apports extérieurs, et l'homme, considéré comme un étant individuel, suppose de nombreux attributs ou propriétés totalement distincts de sa nature.

Une précision s'impose pour ne pas retomber dans le risque d'équivocités langagières précédemment dénoncées. Le terme d'étant revêt deux significations: $1^{\circ}$ ) comme participe du verbe sum correspond au terme «existant» ${ }^{31} ; 2^{\circ}$ ) comme nom substantif du même verbe désignant ce qui possède une nature ou une essence. Il désigne comme nom, l'essence, comme participe, l'existence ${ }^{32}$. On peut prendre pour exemple le terme « vivant » qui est considéré soit en tant que participe du verbe « vivre », c'est-à-dire de l'acte de vivre et non de l'essence du vivant, soit comme un nom substantif du même verbe désignant l'essence du vivant. L'équivocité ne sera par conséquent levée qu'à prendre en charge dans toute exposition métaphysique la division de l'étant considéré nominalement et comme participe. Suárez convie également son lecteur à faire preuve de la même prudence. En effet, l'examen de l'étant dans l'investigation métaphysique requiert la détermination de sa relation aux autres étants, inférieurs et supérieurs. Il est nécessaire d'expliciter le statut de l'ens

28 Mas, Disputación metafísica, Ch. III, n. 26, 427.

29 C'est l'objet de la détermination anthropologique effectuée par Suárez qui n'occupe pas une place centrale dans l'œuvre de Diego Mas.

30 Mas, Disputación metafisica, Livre I, Ch. III, n. 26, 427.

31 Mas, Disputación metafísica, n. 29, 428.

32 Mas, Disputación metafísica, n. 31, 429. 
comme participe et comme nom. La dérivation linguistique de ens à partir de esse est en mesure d'être justifiée à partir du moment où l'on considère corrélativement ens en tant que participe de esse et en tant que nom. ${ }^{33} E n s$, comme participe du verbe esse, désigne l'étant en acte, l'actualité de l'exister qui prend en considération la dimension temporelle propre à la forme verbale. Comme nom, il renvoie à la dissociation de l'essence réelle d'avec l'existence en acte; il exprime l'étant en fonction de l'essence considérée comme possibilité. Pour Suárez, l'enjeu linguistique et ontologique de la provenance du nom ens à partir du verbe esse, s'inscrit dans la conformité à l'héritage métaphysique de la détermination de l'ens en acte et en puissance. Le remodelage de cette distinction récurrente est un préalable à la constitution d'une ontologie ayant pour objectif de repenser le statut de l'étant pour rouvrir la métaphysique à elle-même. Ainsi, la position de l'antériorité du participe sur le nom sera la condition en fonction de laquelle l'essence sera pensée sous la catégorie du possible et l'exister comme espèce du pouvoir-exister, c'est-à-dire la condition de l'étantité généralement considérée.

À la subdivision linguistique de la compréhension de l'étant, il faut également invoquer, pour Diego Mas, afin de préciser l'objet de la métaphysique, ses subdivisions qui scandent la mise en œuvre des conditions de sa connaissance et qui font l'objet d'une analyse systématique dans les Disputes métaphysiques de Suárez. Elles sont au nombre de huit et jalonnent l'histoire de la métaphysique ${ }^{34}$ et ne manquent pas de faire surgir une difficulté à propos de l'ordre à adopter dans ce processus de division, notamment relativement à l'ordre de l'antériorité et de la postériorité. La première concerne la division entre l'étant de raison et l'étant réel établissant l'écart entre ce qui existe par l'entendement et ce qui existe indépendamment de l'intellect. La deuxième correspond à la division entre l'étant par soi et l'étant par accident, la troisième entre l'étant réel et l'étant absolu, la quatrième entre l'étant considéré selon l'unité ou la multiplicité, la cinquième concerne la subdivision de l'étant en trois transcendantaux : l'un, le vrai et le bien, la sixième, entre l'étant fini et infini, la septième entre l'étant en acte et l'étant en puissance, et enfin la huitième, la division de l'étant fini en dix catégories.

Dans l'ordre de la connaissance, il convient de reconnaître pour Diego Mas qu'une division peut être antérieure selon deux modalités : selon la dignité ou la nature. ${ }^{35}$ Conformément à la première, cela signifie que la division s'effectue à 
partir du degré d'éminence reconnu à des classes d'étants ; conformément à la deuxième, cela implique que les éléments qui sont divisés sont en mesure de faire l'objet d'une compréhension immédiate et première. Dès lors, pour Diego Mas, la division de l'étant en étant réel et étant de raison doit être considérée comme première, alors même qu'elle est examinée dans la dernière Dispute métaphysique chez Suárez. Par là est exprimée la division d'une priorité selon la nature parce que ces deux éléments sont contenus immédiatement sous l'étant avant même que l'on puisse établir les autres distinctions. En effet, tout rapport de l'intellect à l'étant ne tire son fondement que de la distinction qui y préside entre ce qui n'a d'existence que par l'intellect et ce qui en a une abstraction faite de la production intellective ; et il y a là comme un prélude à la forme moderne d'une telle distinction qui se dira précisément dans le moment d'autorévélation de l'étant sous la double modalité du sujet et de l'objet. Il n'en reste pas moins qu'une telle division ne saurait prétendre à l'antériorité selon la dignité de l'objet de la connaissance puisque l'étant de raison est le moins éminent de tous les étants.

Or, ce qui est au commencement dans le processus d'une telle détermination chez Diego Mas est à la fin pour Suárez. Bien que ce dernier achève ses Disputes métaphysiques par l'étude des étants de raison portant sur les intentions et les relations logiques, cet examen n'en constitue pas moins l'aboutissement de la division ontologique entre l'étant fini et l'étant infini qui s'avère centrale dans la structuration du raisonnement métaphysique. L'étant de raison revêt une fonction gnoséologique portant à son terme l'exigence ontologique initiale de l'intelligibilité de l'étant, en renouvelant le lien de l'intellect à ses propres productions, ainsi qu'à la réalité ${ }^{36}$. Un tel étant n'a ni existence réelle, ni véritable ressemblance avec les étants réels. En effet, les étants de raison sont « des ombres d'étant $»^{37}$, ce qui signifie qu'ils n'ont pas d'intelligibilité en soi, bien plutôt à partir d'une analogie avec les étants réels. Ils ne possèdent un être objectif que par la raison; par conséquent, les propriétés spécifiques aux étants de raison impliquent d'être objectivement dans l'intellect ou d'être pensé comme étant sans avoir par soi d'entité. La précision apportée par Suárez, par rapport à Diego Mas, lors de son interrogation sur les étants de raison réside dans le fait que cette dernière permet d'établir une différenciation entre la fonction logique et la fonction existentielle du verbe «être ». Se référer à un étant de raison implique, en opposition à l'énonciation existentielle, la

36 Voir Jean-Paul Coujou, "Les êtres de raison et l'extension logique du champ de l'ontologie". En Francisco Suárez, Les êtres de raison. Disputes métaphysique LIV (Paris : Vrin, 2001), pp. 9-44.

37 Coujou, "Les êtres de raison et l'extension logique du champ de l'ontologie", Proême, 47. 
reconnaissance du point de vue logique de l'inexistence de ce qui est posé. L'entendement humain manifeste donc la faculté de faire abstraction de l'existence en tant que fait brut pour instaurer une forme d'être à partir de l'inexistant.

L'emploi légitime des étants de raison requiert pour Suárez de déterminer les moments opportuns pour s'y référer. Ainsi, $1^{\circ}$ ) la création des étants de raison résulte de l'exigence intellectuelle d'accession à la connaissance de principes explicatifs de la relation entre les étants réels comme on peut le constater, notamment dans les domaines de la logique ou des mathématiques. La mise en place d'une conceptualisation de la négation ou de la privation découle par exemple d'une opération de l'intellect instaurant un nouveau mode d'être, très précisément celui des étants de raison. $2^{\circ}$ ) L'intellect engendre au moyen du langage des relations de raison ayant fonction de substituts en l'absence de relations réelles. Par conséquent, dans l'investigation métaphysique l'étant de raison revêt un rôle cognitif permettant de procéder à une extension logique du champ du savoir.

La considération du questionnement initial de la métaphysique chez Diego Mas et Suárez permet, à partir de ces remarques, de reformuler l'interrogation sur le sens de ce qui est en l'émancipant d'une hiérarchisation fondatrice des différentes manifestations de l'étant. En effet, pour Diego Mas, l'étant se dit uniquement de manière homonyme de Dieu et des créatures ${ }^{38}$. Il n'y a aucune proportion entre les deux et, par conséquent, aucune analogie (car pour qu'il y ait analogie, il faut qu'intervienne une certaine ressemblance), comme il apparaît manifeste entre un étant fini et un étant infini, seulement une homonymie qui est le signe d'une prédication selon le nom et non selon la raison. Pour Suárez, dès lors que pour une refondation de la métaphysique Dieu n'est plus en mesure de constituer un point de départ, il faut premièrement afin de se donner un objet adéquat, expliciter le statut de l'étant et ce que signifie universellement « être », ce qui suppose précisément de se rapporter aux raisons communes de l'étant ${ }^{39}$ ou de promouvoir les conditions et le contenu d'une ontologie transcendantale assurant la fondation objective de l'universel. La voie est dès lors ouverte pour une redéfinition de la fonction, de la finalité et de l'utilité de la métaphysique ${ }^{40}$. 
Avec les œuvres de Diego Mas et Suárez, un projet commun est affirmé à partir de la mutation décisive des concepts ontologiques légués par la tradition et consacrée par l'avènement d'une métaphysique transcendantale recentrant le processus de la production du savoir sur l'universel. Le point de départ d'un renouvellement du questionnement métaphysique requiert l'examen systématique de son historicité ce qui explique précisément que s'il est impossible de faire l'économie de l'héritage fondateur d'Aristote, il est désormais nécessaire de reconnaître que l'histoire de la métaphysique ne saurait s'y réduire. On ne peut pas poursuivre sans Aristote, on ne peut pas poursuivre simplement avec.

Néanmoins, si le renouveau de la métaphysique consiste également dans la prise en considération de son histoire, comment faire pour que cela n'abolisse pas toute espèce de fondement ? Il faut que la métaphysique se rapporte, malgré l'historicité du savoir et du rapport historique de la raison à l'étant, à l'intemporalité de l'universel, celle de l'étant en tant qu'étant réel comme condition de possibilité du commencement de tout savoir. Or, cela s'effectue au prix d'un paradoxe non négligeable: si l'universel prévaut par rapport au premier, ainsi que le confirme le dépassement dans l'ordre de la production du savoir de la priorité de l'étant infini sur l'étant fini, il deviendrait en excédant le premier, lui-même premier, même si l'ontologie transcendantale en se substituant à la dénomination de philosophie première veut s'en prémunir. Pour Diego Mas et Suárez, et tel serait leur apport spécifique, le caractère transcendantal de l'ontologie s'impose par rapport à la priorité ontique du premier, sans pour autant renvoyer à sa convertibilité comme condition du pensable à une quelconque priorité ontique. C'est bien dans une logique de la reconstruction que doit être pensé le destin de la métaphysique selon les deux auteurs.

\section{REFERENCES BIBLIOGRAPHIQUES}

Alexandre d'Aphrodise, Quaestiones naturales et morales ..., Venetiis: s.e. 1546. Philoponus, Ioannes Aristotelis de anima libri tres ..., Lugduni: s.e. 1544.

Báñez, Domingo. Scolastica commentaria in primam partem ... D. Thomae usque ad LXIII quaestionem complectentia. Venetiis: 1585.

Coujou, Jean-Paul. "Les êtres de raison et l'extension logique du champ de l'ontologie". En Francisco Suárez, Les êtres de raison. Disputes métaphysique LIV. Paris: Vrin, 2001, 9-44.

Iavellus, Crysostomus. Tractatus de Transcendentibus previus methaphisice. s.e.: 1528 . 
Mas, Diego. Disputación metafisica sobre el ente y sus propiedades (1587), Traducción y introducción de J. Gallego Salvadores. Pamplona: EUNSA, 2003.

Pereira, Benedictus. De communibus omnium rerum naturalium principiis. Romae: s.e., 1562.

Philopon, Jean. Commentaire sur le traité "De l'âme» d'Aristote (vers 510-515), édition par Michael Hayduck, In Aristotelis « De Anima » libros commentaria. Berlin: De Gruyter 1897.

Pic de la Mirandole, Jean. L'être et l'un in Euvres philosophiques. Paris: PUF, 1995

Simplicius, De anima, édition par Michael Hayduck, In Aristotelis «De anima », coll. « Commentaria in Aristotelem Graeca » (CAG), t. XI. Berlin: De Gruyter, 1882.

Simplicius. In libros de Anima Aristotelis, interprete I. Fasseolo Patavino.Venetiis: s.e. 1543.

Suárez, Franciscus. Metaphysicorum Disputationum, in quibus et universa naturalis theologia ordinate traditur, et quaestiones omnes ad duodecim Aristotelis libros pertinentes accurate disputantur. Tomus prior. Salmanticae: apud Ioannem et Andream Renaut fratres, 1597.

Suárez, Franciscus. Opera Omnia, Volume 25, In Metaphysicam Aristotelis. Disputationes metaphysicae, (I-XXVII). Paris: Vives, 1856.

Suárez, Franciscus. Opera Omnia, Volume 26, Disputationes metaphysicae (XXVIII-LIV). Paris: Vives 1856.

Jean Paul Coujou Institut catholique de Toulouse 31, rue de la Fonderie, BP 7012, 31068 Toulouse (Francia) https://orcid.org/0000-0003-4203-2652 